\title{
Two-Dimensional Reconstruction of Heat Transfer in a Flat Flame Furnace through Computer-Based Tomography and Tunable-Diode-Laser Absorption Spectroscopy
}

\author{
Xiaoyong Wang* \\ Huainan Union University, Huainan, 232038, China \\ *Corresponding Author: Xiaoyong Wang. Email: A4050550221@163.com \\ Received: 31 December 2019; Accepted: 23 July 2020
}

\begin{abstract}
To explore the inherent characteristics of combustion-induced heat transfer in a flat flame furnace, a sophisticated hybrid method is introduced by combining a computer-based tomography (CT)-algebraic iterative algorithm and Tunable Diode Laser Absorption Spectroscopy (TDLAS). This technique is used to analyze the distribution of vapor concentration and furnace temperature. It is shown that by using this strategy a variety of details can be obtained, which would otherwise be out of reach.
\end{abstract}

Keywords: Computed tomography algorithm; flame furnace; TDLAS; temperature; boundary

\section{Introduction}

Along with the continuous consumption of fossil energy worldwide, the available natural resources are constantly decreasing; thus, increasing the utilization and development of fossil energy resources are receiving wide attention globally [1,2]. In China, it is particularly critical due to its large population. Although the total resources are abundant in China, the per capita possession is much lower than the world average levels. Therefore, China has also increased the specific development and utilization of fossil energy resources [3]. Combustion is the major manifestation of fossil energy. At present, the research on heat transfer during the combustion of fossil energy is at an early stage. The major research direction is focused on the control and management of combustion efficiency, which is from the macro perspective. However, there is no in-depth study on the characteristics of the heat transfer in combustion of fossil resources from the micro perspective, which is a significant research direction [4]. Through the research on the combustion heat transfer of fossil energy, the efficiency of fossil energy, such as coal, can be greatly improved. Not only can it save resources but also significantly reduce pollution to the global environment. Through continuous research and development, the heat transfer of fossil energy during combustion will be widely applied for daily purposes in the future. Whether for the military, aerospace, industrial, or daily purposes, the use of combustion energy can become the driving force for processing and production $[5,6]$. Research on the microscopic characteristics of the combustion heat transfer of fossil energy requires sustained high-level talents and economic investment to achieve excellent results [7]. At present, there are many problems involved in utilizing fossil energy resources, such as instability, safety, 
and low efficiency. These difficulties currently hinder the in-depth utilization of fossil energy resources [8]. Some researchers have used direct absorption spectrum time division multiplexing technology and tunable diode laser absorption spectroscopy (TDLAS) technology to measure the temperature and component concentration of flat flame furnace, and achieved good experimental results $[9,10]$. Therefore, this study considers the advantages and disadvantages of traditional resource utilization. The two-dimensional reconstruction of the distribution of combustion temperature and concentration of fossil energy in flat flame furnace is carried out by using the hybrid method of computed tomography (CT)-algebraic iteration algorithm and TDLAS. The qualitative and quantitative analysis of the distribution results of combustion temperature and concentration of fossil energy is realized.

In this study, a two-dimensional reconstruction of vapor concentration and furnace temperature in the surface combustion of a flat flame furnace is analyzed and researched by constructing a hybrid method based on CT-algebraic iterative algorithm and TDLAS. Based on the hybrid method of the CT-algebraic iterative algorithm and TDLAS, the data and distribution laws of the concentration and temperature control of the flat flame furnace are investigated. At present, the two-dimensional reconstruction of the combustion process of flat flame furnace is more focused on the exploration of a single reconstruction method, based on this, CT algebraic iterative algorithm and TDLAS algorithm are fused innovatively in experiment. The purpose of study is to provide some experimental support for the follow-up study of the algebraic iterative algorithm of $\mathrm{CT}$ and the further study of the combustion process of the flat flame furnace.

\section{Methodology}

\subsection{Measurement Principles}

In this experiment, TDLAS temperature concentration measurement principle and algebraic iterative reconstruction method are combined to measure the temperature and water vapor concentration of flat flame furnace. The purpose is to reconstruct the temperature field and water vapor concentration distribution in combustion area. The Beer-Lambert Law: The principle of tunable semiconductor laser absorption spectroscopy is based on the Beer-Lambert Law, i.e.,

$\frac{\mathrm{I}_{\mathrm{t}}(\mathrm{v})}{\mathrm{I}_{0}(\mathrm{v})}=\exp \left(-\mathrm{k}_{\mathrm{v}} \mathrm{L}\right)$

where $I_{0}(v)$ is the incident light intensity $(\mathrm{mW})$, and $I_{t}(v)$ is the transmitted light intensity $(\mathrm{mW}) . \mathrm{k}_{\mathrm{v}}$ is the absorption coefficient, $\mathrm{L}$ is the length of light through the medium.

$\mathrm{k}_{\mathrm{v}}=\mathrm{px}_{\mathrm{abs}} \mathrm{S}_{\mathrm{i}}(\mathrm{T}) \Phi_{\mathrm{v}}$

where $\mathrm{P}$ is the total static pressure (atm) of the mixed gas, and $x_{a b s}$ is the volume concentration of the absorbed gas. $\mathrm{S}_{\mathrm{i}}(\mathrm{T})$ and $\Phi_{\mathrm{v}}$ denote the line strength and area normalized line function of molecular transition spectrum of matter. According to Eqs. (1) and (2), the absorbance $A(v)$ and integral absorbance A can be obtained as follows:

$A(v)=\operatorname{In}\left(\frac{I_{0}}{I_{t}}\right)$
$A_{i}=\int \operatorname{In}\left(\frac{I_{0}}{I_{t}}\right) d v=p_{a b s} S_{i}(T) L$

where the integral absorbance Ai can be expressed as the product of the gas absorption coefficient $\alpha_{v}$ and the absorption path length L; thus: 
$\alpha_{\mathrm{v}}=\mathrm{px}_{\mathrm{abs}} \mathrm{S}_{\mathrm{i}}(\mathrm{T}) \mathrm{L}$

For a specific gas and selected spectral line, the line strength $S(T)$ is only temperature-dependent, which can be expressed as:

$$
\mathrm{S}(\mathrm{T})=\mathrm{S}\left(\mathrm{T}_{0}\right) \frac{\mathrm{Q}\left(\mathrm{T}_{0}\right)}{\mathrm{Q}(\mathrm{T})} \frac{\mathrm{T}_{0}}{\mathrm{~T}} \exp \left[-\frac{\mathrm{hcE} \mathrm{E}^{\prime \prime}}{\mathrm{k}}\left(\frac{1}{\mathrm{~T}}-\frac{1}{\mathrm{~T}_{0}}\right)\right] \times\left[1-\exp \left(\frac{-\mathrm{hcv}_{0}}{\mathrm{kT}}\right)\right]\left[1-\exp \left(\frac{-\mathrm{hcv}_{0}}{\mathrm{kT}_{0}}\right)\right]^{-1}
$$

where $S\left(T_{0}\right)$ is the line strength of the absorption spectral line to be measured at the reference temperature $T_{0}$, while $\mathrm{Q}(\mathrm{T})$ and $\mathrm{Q}(\mathrm{T} 0)$ are the partition functions of the measured gas $\mathrm{T}$ and $\mathrm{T} 0$, respectively. $\mathrm{E}^{\prime \prime}$ represents low-level energy, $\mathrm{h}$ is Planck's constant, and $\mathrm{k}$ is Boltzmann's constant.

\subsection{Measurement Principles of TDLAS Temperature and Concentration}

The absorption line strength ratio method usually selects two absorption lines of the same gas molecule, and the internal partition function $\mathrm{Q}(\mathrm{T})$ can be eliminated by the absorption line strength ratio, thereby:

$$
\mathrm{R}(\mathrm{T})=\frac{\mathrm{S}_{1}(\mathrm{~T})}{\mathrm{S}_{2}(\mathrm{~T})}=\frac{\mathrm{S}_{1}\left(\mathrm{~T}_{0}\right)}{\mathrm{S}_{2}\left(\mathrm{~T}_{0}\right)} \exp \left[-\frac{\mathrm{hc}}{\mathrm{k}}\left(\mathrm{E}_{1}^{\prime \prime}-\mathrm{E}_{2}{ }^{\prime \prime}\right)\left(\frac{1}{\mathrm{~T}}-\frac{1}{\mathrm{~T}_{0}}\right)\right]\left[1-\exp \left(\frac{-\mathrm{hcv}_{01}}{\mathrm{kT}}\right)\right]\left[1-\exp \left(\frac{-\mathrm{hcv}_{02}}{\mathrm{kT}_{0}}\right)\right]^{-1}
$$

For the case where the absorption line is not very different in wavelength, the ratio of the stimulated radiation correction term in the ratio is Approximately 1, which is usually ignored and simplified as:

$\mathrm{R}(\mathrm{T})=\frac{\mathrm{S}_{1}(\mathrm{~T})}{\mathrm{S}_{2}(\mathrm{~T})}=\frac{\mathrm{S}_{1}\left(\mathrm{~T}_{0}\right)}{\mathrm{S}_{2}\left(\mathrm{~T}_{0}\right)} \exp \left[-\frac{\mathrm{hc}}{\mathrm{k}}\left(\mathrm{E}_{1}^{\prime \prime}-\mathrm{E}_{2}^{\prime \prime}\right)\left(\frac{1}{\mathrm{~T}}-\frac{1}{\mathrm{~T}_{0}}\right)\right]$

Therefore, the line strength ratio is a function of the temperature $\mathrm{T}$, and $\mathrm{T}$ can be deduced by measuring $\mathrm{R}$. Substituting $\mathrm{T}$ into the equation to find the line strength at the corresponding temperature, the water vapor concentration can be obtained.

\subsection{CT Reconstruction Algorithm-Algebraic Iterative Algorithm}

The ART algorithm discussed in this study is a kind of critical CT algorithm. Its essence is to solve linear equations, and the number of unknowns in the equations is greater than the number of equations $[11,12]$. By adding prior information and constraints, the suitable solutions to the equations are searched, and the principle of absorption spectroscopy is shown in Fig. 1.

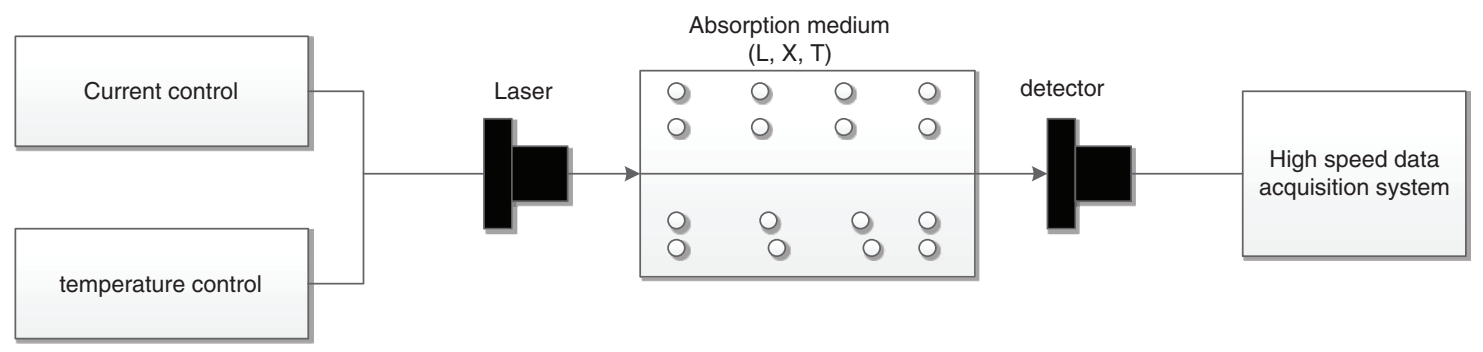

Figure 1: Principle of absorption spectroscopy

The flow of the CT-algebra iterative algorithm is shown in Fig. 2. It is mainly reflected in the following aspects. The first is to reprocess the area to be reconstructed, and the core is to make it discrete into grids with a certain resolution [13]. The second is to reconstruct the projection equations of all rays and have all the 


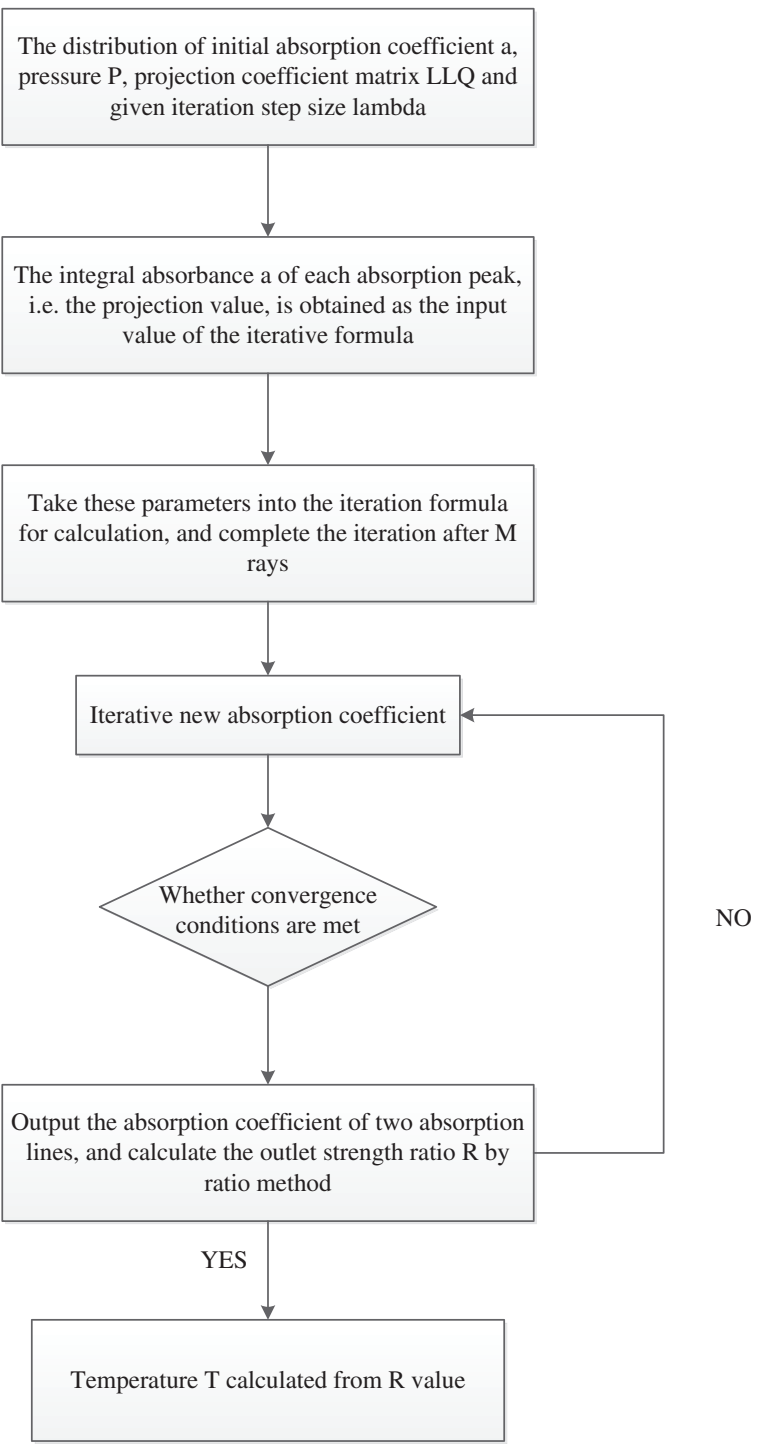

Figure 2: Flowchart of CT-algebraic iterative algorithm

equations be mixed according to certain rules, forming a characteristic linear equation. Each mixed equation contains the attenuation coefficient of the equation [14]. The third is to re-assign values for the grids that are discretely set in the first step. Then, the numerical calculation of each projection is performed according to the re-assigned value, which must be strict [15]. The fourth is to calculate the difference between the real value and the projection value according to the projection results obtained by the first projection calculation equation. Then, the values that have been obtained are re-corrected according to the iterative equation of CT algebra to obtain the accurate grid values. The fifth is to calculate the equation and bring it into the next projection according to the corrected and calculated gray value [16]. Afterward, the same operation steps are performed according to the fourth step, and the last projection equation is continued to complete the entire iteration process. The next step is to start the loop operation. The second iteration process is started from the first projection equation. After several loop iterations, the difference between the values of the adjacent grids is very small. Thus, the calculation step is completed to obtain the result value of the linear equation [17]. It is the flow of the entire CT-algebraic iterative algorithm. The real process is 
strictly prohibited and efficient. The obtained results need to go through countless iterations before the expected results are obtained. Based on relevant data and information, this study has performed various processes and manipulations of the CT-algebra iterative algorithm, including redesigning and improving the smoothing criterion, adding non-negative numerical identification and modification, and performing self-corrections and updates of the relaxation factor of the algorithm. Such operations improve not only the stability of the algorithm but also the accuracy and speed of the operation, thereby increasing the rationality of the operation of the equation [18].

The basic principle of CT: According to the Beer-Lambert absorption law in TDLAS technology, when a laser with an intensity of $\mathrm{I} 0$ is irradiated on a gas medium with an attenuation coefficient of $\mathrm{u}$, after passing the distance 1 , if the output light intensity is set to 1 , the relationship between the incident light and the incident light is:

$\mathrm{I}_{\mathrm{t}}=\mathrm{I}_{0} \mathrm{e}^{-\mathrm{ul}}$

If the density across the medium is non-uniform, the path 1 needs to be discretized, and fn is the discretization attenuation coefficient. At this time, the projection $p$ in a certain direction is defined as:

$\mathrm{p}=\left(\mathrm{f}_{1}+\mathrm{f}_{2}+\mathrm{f}_{3}+\mathrm{f}_{4} \ldots . .+\mathrm{f}_{\mathrm{n}}\right) \Delta \mathrm{l}=\sum_{\mathrm{i}=1}^{\mathrm{n}} \mathrm{f}_{\mathrm{i}} \Delta \mathrm{l}=\operatorname{In} \frac{\mathrm{T}_{0}}{\mathrm{~T}}$

when $\Delta l$ tends to infinite hours, the above equation can be written in the integral form:

$p=\int_{1} f(1) d l$

It represents the projection of the attenuation coefficient $\mathrm{f}(\mathrm{l})$ along the 1 direction. If the projection data $\mathrm{p}$ at different angles and positions is known, the attenuation coefficient $f(l)$ can be inferred through a series of projection values by a reconstruction algorithm. This process is called image reconstruction, i.e., CT. A typical experimental setup of the fixed wavelength direct absorption method is shown in Fig. 3, which is extended to the field of laser absorption spectroscopy. If the integral absorption rate at the selected absorption line and different position angles is obtained in the experiment, the two-dimensional distribution of combustion temperature and concentration can be measured by the reconstruction algorithm.

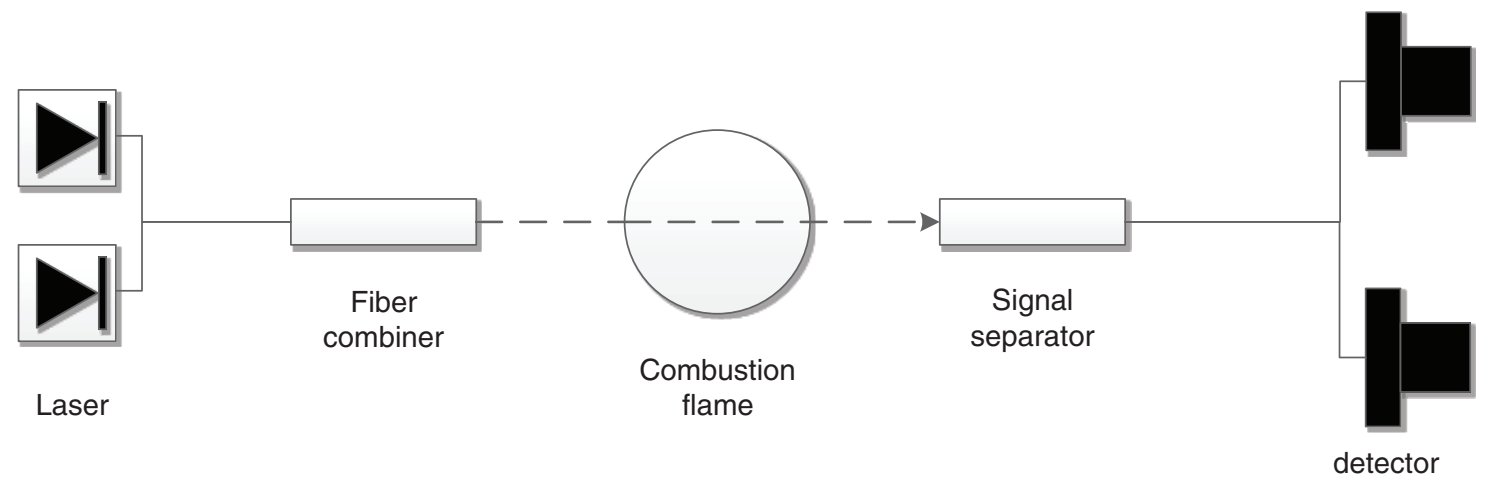

Figure 3: Typical experimental setup of fixed wavelength direct absorption method 


\subsection{Algorithm Reconstruction}

To obtain a two-dimensional distribution image of combustion temperature and concentration, the reconstruction area is first divided into $\mathrm{J}(\mathrm{J}=\mathrm{MXN})$ grids. Meanwhile, the gas parameters, such as temperature, pressure, and concentration, inside the grid are considered to be uniform. When a certain laser beam i passes through the target area, the integral absorbance of the absorption line with the center frequency vi can be discretized as:

$\mathrm{A}_{\mathrm{vi}, \mathrm{i}}=\sum_{\mathrm{n}=1}^{\mathrm{N}} \sum_{\mathrm{m}=1}^{\mathrm{M}}[\operatorname{PXS}(\mathrm{T})]_{\mathrm{vi},(\mathrm{m}, \mathrm{n})} \mathrm{L}_{\mathrm{i},(\mathrm{m}, \mathrm{n})}=\sum_{\mathrm{n}=1}^{\mathrm{N}} \sum_{\mathrm{m}=1}^{\mathrm{M}} \alpha_{\mathrm{vi},(\mathrm{m}, \mathrm{n})} \mathrm{L}_{\mathrm{i},(\mathrm{m}, \mathrm{n})}$

The above equation is also called the gas absorption equation, where I is the total number of laser beams, $\mathrm{m}$ and $\mathrm{n}$ are respectively the row and column indexes of the image matrix $\mathrm{MXN}$, and the algorithm optical path is shown in Fig. 4.

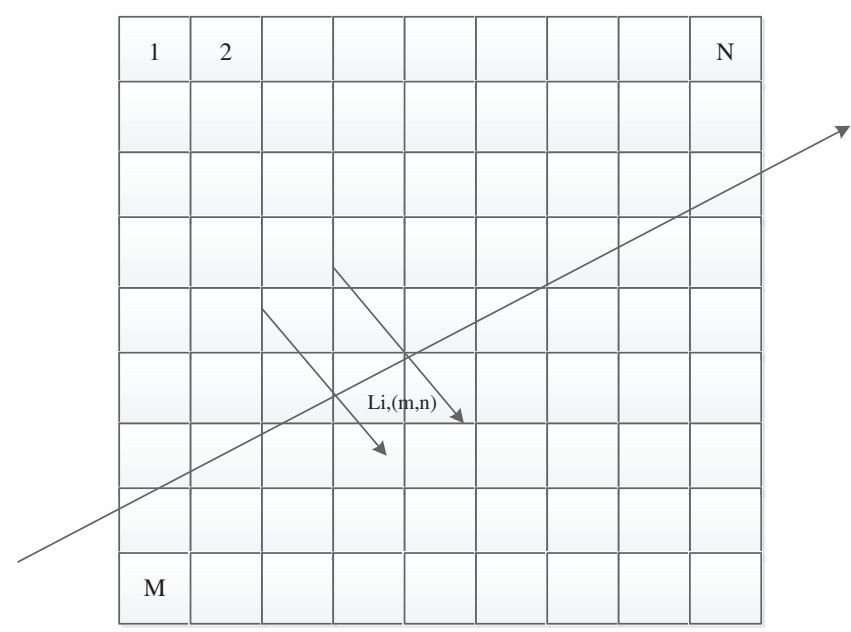

Figure 4: Optical path of the algorithm

When the reconstruction area is scanned by multiple beams at multiple angles and directions, each laser beam has the described projection equation, which is a linear function of pressure, concentration, line intensity, and optical path. By combining these equations, a linear system of equations is obtained, as shown in the following equation:

$\left\{\begin{array}{c}\mathrm{A}_{1}=\mathrm{L}_{1,(1,1)} \alpha_{(1,1)}+\mathrm{L}_{2,(1,2)} \alpha_{(1,2)}+\ldots+\mathrm{L}_{1,(\mathrm{~m}, \mathrm{n})} \alpha_{(\mathrm{m}, \mathrm{n})}+\ldots+\mathrm{L}_{1,(\mathrm{M}, \mathrm{N})} \alpha_{(\mathrm{M}, \mathrm{N})} \\ \mathrm{A}_{2}=\mathrm{L}_{2,(1,1)} \alpha_{(1,1)}+\mathrm{L}_{2,(1,2)} \alpha_{(1,2)}+\ldots+\mathrm{L}_{2,(\mathrm{~m}, \mathrm{n})} \alpha_{(\mathrm{m}, \mathrm{n})}+\ldots+\mathrm{L}_{2,(\mathrm{M}, \mathrm{N})} \alpha_{(\mathrm{M}, \mathrm{N})} \\ \mathrm{A}_{\mathrm{i}}=\mathrm{L}_{\mathrm{i},(1,1)} \alpha_{(1,1)}+\mathrm{L}_{\mathrm{i},(1,2)} \alpha_{(1,2)}+\ldots+\mathrm{L}_{\mathrm{i},(\mathrm{m}, \mathrm{n})} \alpha_{(\mathrm{m}, \mathrm{n})}+\ldots+\mathrm{L}_{\mathrm{i},(\mathrm{M}, \mathrm{N})} \alpha_{(\mathrm{M}, \mathrm{N})}\end{array}\right.$

\subsection{Temperature and Concentration Reconstruction under Numerical Simulation}

The schematic diagram of the flat flame furnace one-dimensional test system is shown in Fig. 5. Before the experiment, numerical simulation calculations are performed. The reconstruction area is a square with equal length and width, which is divided into 36 grids with an area of $6 \times 6$. The laser beam has 8 rays in the horizontal and vertical directions. The temperature distribution along the two assumed original grids is $900 \mathrm{k}$ in the middle 9 grids, and the vapor concentration is 0.2 . The temperature in the outer 16 grids is $600 \mathrm{k}$, and the vapor concentration is 0.2 . After statistical analysis of the data, the maximum deviation between the temperature reconstruction result and the original temperature distribution is 


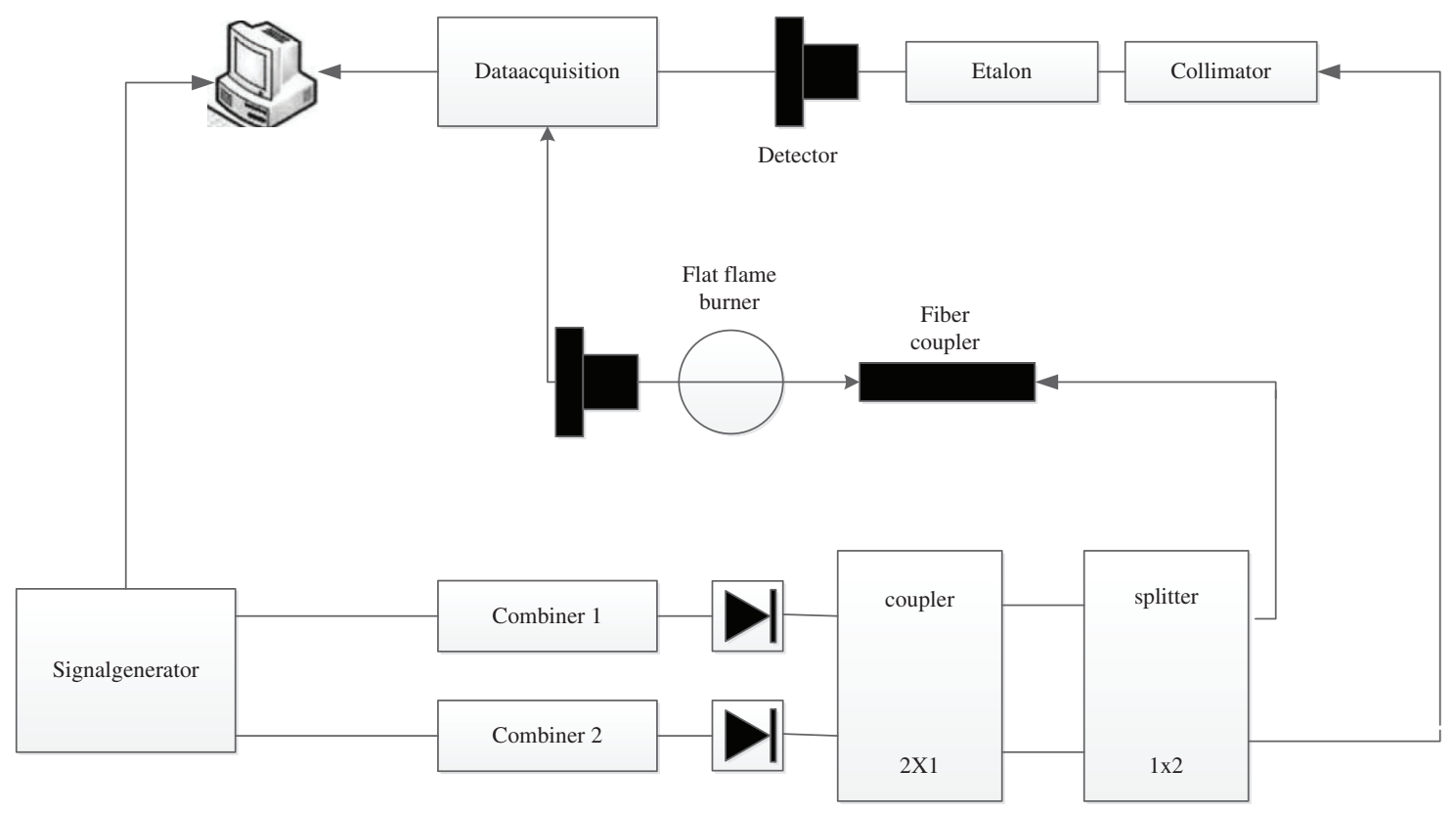

Figure 5: Schematic diagram of the one-dimensional test system for flat flame furnace

$0.93 \%$, while the maximum deviation between the vapor concentration reconstruction result and the original concentration distribution is $0.71 \%$, in which the corresponding noise is also added for reconstruction.

In terms of CT-algebraic iterative algorithms, its functions are rich and powerful. It not only achieves the reconstruction and setting of projection equations but also has high efficiency and accuracy, which is critical for practical applications [19]. However, for the projection reconstruction equations with high-scoring requirements, the entire iterative calculation process is huge, the time is long, and the accuracy of the results will also be affected. Due to such a problem, this study has improved and processed the algorithm to propose a matrix method for coefficient calculation with a relatively fast calculation speed, which simplifies and organizes the traditional coefficient calculation process and greatly reduces the engineering volume and time of the operation [20]. Based on relevant data and information, this study has performed various processes and manipulations of the CT-algebra iterative algorithm, including redesigning and improving the smoothing criterion, adding non-negative numerical identification and modification, and performing self-corrections and updates of the relaxation factor of the algorithm. Through such operations, not only the stability of the algorithm is improved, but also the accuracy and speed of the operation. Multiple pixels of the grid are processed and modified. Regarding the reconstruction process of the water vapor concentration of the flame furnace, the previously lagging With the method of rebuilding the temperature and then obtaining the results, the method was abandoned, and a new multiple iteration process was used to directly reconstruct the water vapor concentration and build the process [21]. The results obtained through this method are accurate and reliable. Then, the algorithm for verification and improvement is directly transferred to the reconstruction process of the double peaks. The results obtained are also accurate and reliable. In the process of improving and researching the accuracy [22].

\subsection{Reconstruction Accuracy Analysis}

The results of the combustion field distribution reconstruction are affected by a variety of factors, among which the most significant include the number of projection directions, the number of projected rays, and the size of the noise circle [23]. The quality of image reconstruction can be evaluated by relevant criteria. The impact of different factors on the quality of image reconstruction is mainly determined, and the optimal 
reconstruction scheme is given to obtain the optimal reconstruction results. Analysis of the impact of the number of projection directions on the reconstruction accuracy: To evaluate the quality of image reconstruction, the difference between the reconstructed image and the original image is used to evaluate the reconstruction effect. Three error evaluation criteria are given here, i.e.,

Normalized mean square distance criterion D:

$\mathrm{D}=\sqrt{\frac{\sum_{\mathrm{j}=1}^{\mathrm{J}}\left(\mathrm{r}_{\mathrm{j}}-\mathrm{o}_{\mathrm{j}}\right)^{2}}{\sum_{\mathrm{j}=1}^{\mathrm{J}}\left(\mathrm{o}_{\mathrm{j}}-\overline{\mathrm{o}}\right)^{2}}}$

Normalized absolute distance criterion R:

$\mathrm{D}=\frac{\sum_{\mathrm{j}=1}^{\mathrm{J}}\left|\mathrm{o}_{\mathrm{j}}-\mathrm{r}_{\mathrm{j}}\right|}{\sum_{\mathrm{j}=1}^{\mathrm{J}} \mathrm{o}_{\mathrm{j}}}$

Normalized percent error:

error $=\sqrt{\frac{\sum_{j=1}^{J}\left(\frac{r_{j}-o_{j}}{o_{j}}\right)^{2}}{J^{2}}}$

where $o_{j}$ and $r_{j}$ represent the pixel values of pixel $\mathrm{j}$ in the original field distribution and the reconstructed field distribution, $\bar{o}$ indicates the average of the original image pixel values, respectively, and the image matrix has a total of $\mathrm{J}$ pixels. The significance of each criterion parameter is different. The normalized mean square criterion represents a large reconstruction deviation at a small number of points. The normalized absolute distance criterion represents a large number of points that produce small errors. The importance of large errors is not emphasized. The normalized percent error reflects the reconstruction bias produced by each pixel on average. A typical scanning wavelength direct absorption spectroscopy technology experimental system is shown in Fig. 6.

\subsection{Introduction to the Experimental System}

Hardware composition: The hardware components used in the experiment include rotating stage, translation stage, DFB laser, laser drive, self-focusing lens fiber collimator, fiber beam splitter, fiber combiner, photodetector, amplifier circuit board, time division multiplexed circuit board, etalon, and data acquisition and processing system [24]. The two-dimensional reconstruction system of the combustion flow field based on TDLAS technology is shown in Fig. 7.

Software program design: Before setting up a combustion flow field experimental measurement system, the acquisition program and the spectral data processing software are written in Labview. The options mainly include data acquisition average times, sampling rate, sampling length, channel settings, save path, and signal display [25]. 


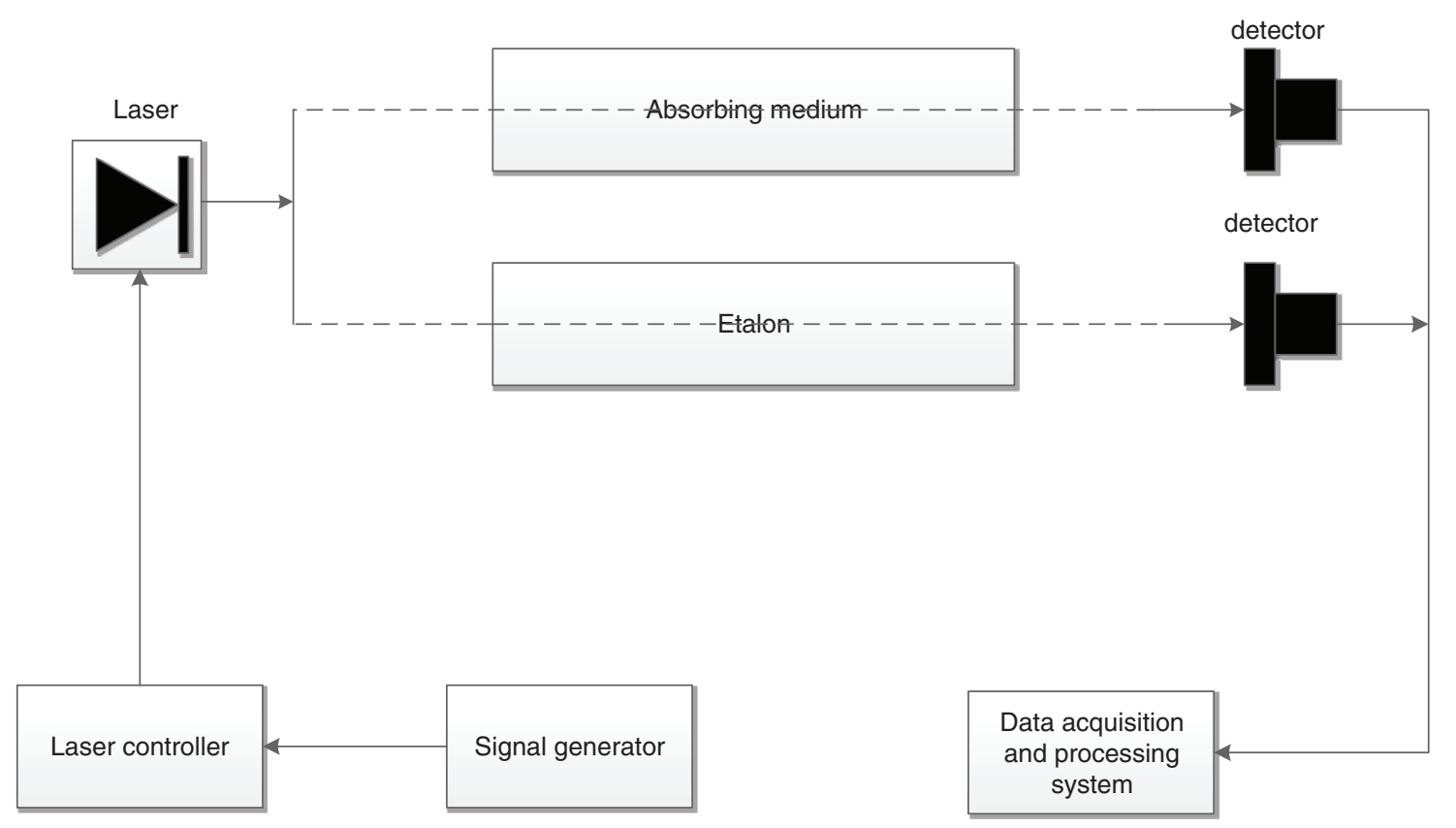

Figure 6: Typical scanning wavelength direct absorption spectroscopy technology experimental system

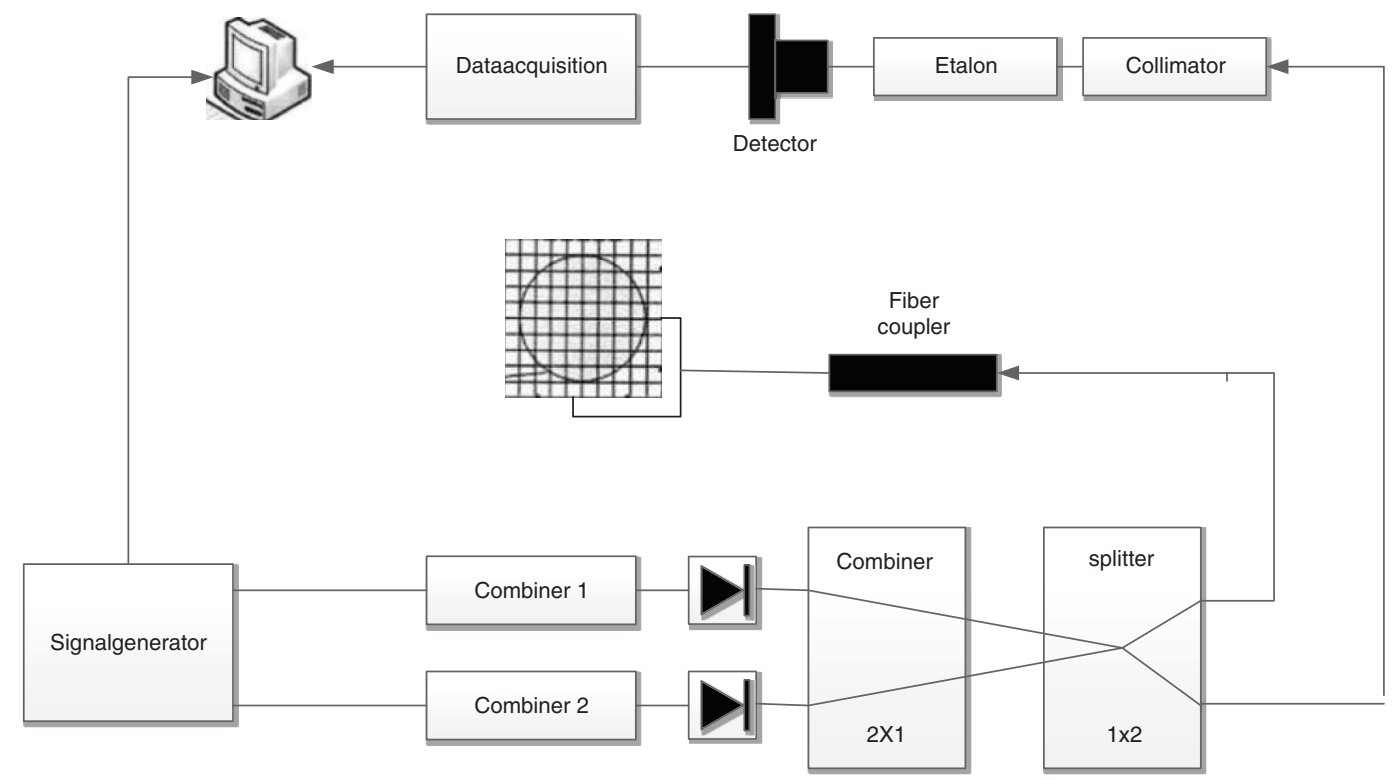

Figure 7: Two-dimensional reconstruction system of combustion flow field based on TDLAS technology

\section{Results and Discussion}

\subsection{The Influence of the Number of Parallel Rays in One View on the Reconstruction Accuracy}

The effect of the number of parallel rays on the reconstruction accuracy in a single angle of view: The number of parallel rays in a single angle of view has a significant effect on the environment for reconstruction. Therefore, the number of projections is set to 20 and remains unchanged. The number of set parallel light is circulated from 11 to 30 according to a certain difference; then, the value of the reconstruction result is calculated and analyzed under different settings to determine whether its accuracy 
is affected and whether it can meet the needs. As shown in the obtained numerical results, with the continuous increase in the number of parallel lights, the furnace temperature distribution and the vapor concentration distribution are analyzed to reconstruct the process. The results show that whether it is the judgment index mean square distance, absolute distance, or percentage error, it shows a slowly decreasing process, i.e., as the number of parallel rays increases, the accuracy of the reconstruction is greatly affected, and the accuracy value is decreasing. The influence of noise on the reconstruction accuracy is quite significant. Therefore, the number of projections is set to 20 and remains unchanged. The number of set different noise levels is circulated from 11 to 30 according to a certain difference; then, the value of the reconstruction result is calculated and analyzed under different settings to determine whether its accuracy is affected and whether it can meet the needs. As shown in the obtained numerical results, with the continuous increase in the noise, the furnace temperature distribution and the vapor concentration distribution are analyzed to reconstruct the process. The results show that whether it is the judgment index mean square distance, absolute distance, or percentage error, it shows a slowly decreasing process, i.e., as the noise increases, the accuracy of the reconstruction is greatly affected, and the accuracy value is decreasing.

\subsection{Two-dimensional Reconstruction Results of Temperature and Concentration of Combustion Heat}

\section{Transfer on Flat Flame Furnace Surface}

The average temperature measured along the $\mathrm{X}$ and $\mathrm{Y}$ directions based on the two-line method is shown in Fig. 8. As shown in the data and trend graphs in the figure, the measured temperature results at the boundary of the flat flame furnace are either on the X-axis or the Y-axis, which are lower than the temperature of the central position of the furnace. Also, the measurement temperatures of the central positions are relatively stable and close to each other. The temperature of the eighth detection optical path in the X-axis direction is slightly higher. Therefore, based on the TDLAS technology and CT-algebraic iterative reconstruction algorithm, the measurement of the surface combustion heat temperature of a flat flame furnace is consistent with the actual results, and the performance is stable and prominent.

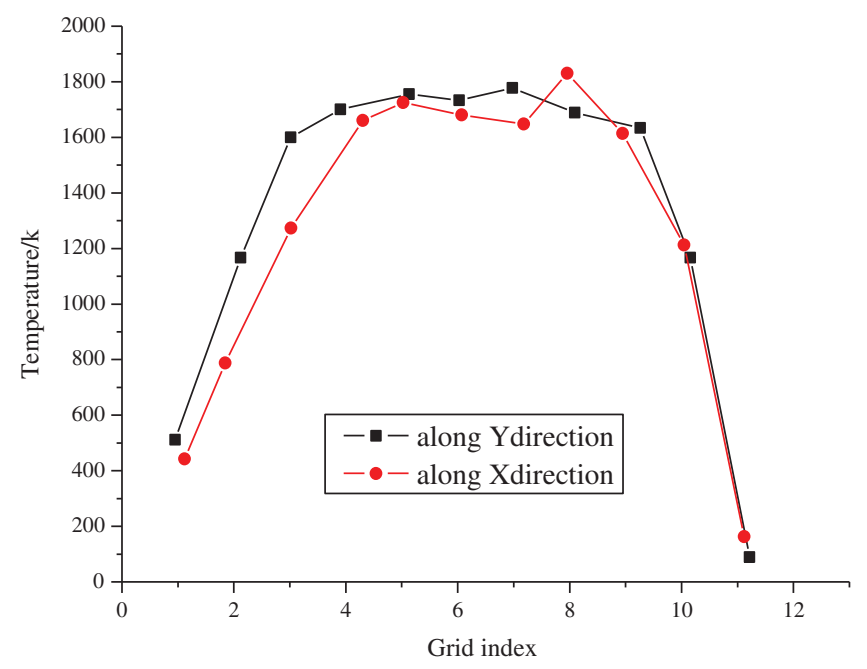

Figure 8: Average temperature measured along the $\mathrm{X}$ and $\mathrm{Y}$ directions based on the two-line method

To eliminate the accidental error and calculate the projection data, the average value is obtained through multiple measurements. Before rotating the rotary table, continuously measure and save multiple sets of data of five signals in this state, then rotate the rotary table for 90 degrees anticlockwise, and repeat the above 
measurement. The rotating time of the rotary table is about 10 seconds, and it takes 3 minutes to collect and process 10 laser signals twice. Fig. 9 shows the integral absorbance data of three signals in the vertical direction for a period without rotation, in which the data points with too large fluctuation are eliminated. As shown in the data and the trend graphs in the figure, the integral absorbance of the 3 sets of data has remained basically stable, while the fluctuation has basically remained within a certain range. It also shows the measurement positions of different levels and different optical paths. The algebraic iterative reconstruction algorithm based on TDLAS technology and CT is suitable for the measurement of combustion heat transfer on the surface of a flat flame furnace. The data is authentic and reliable.

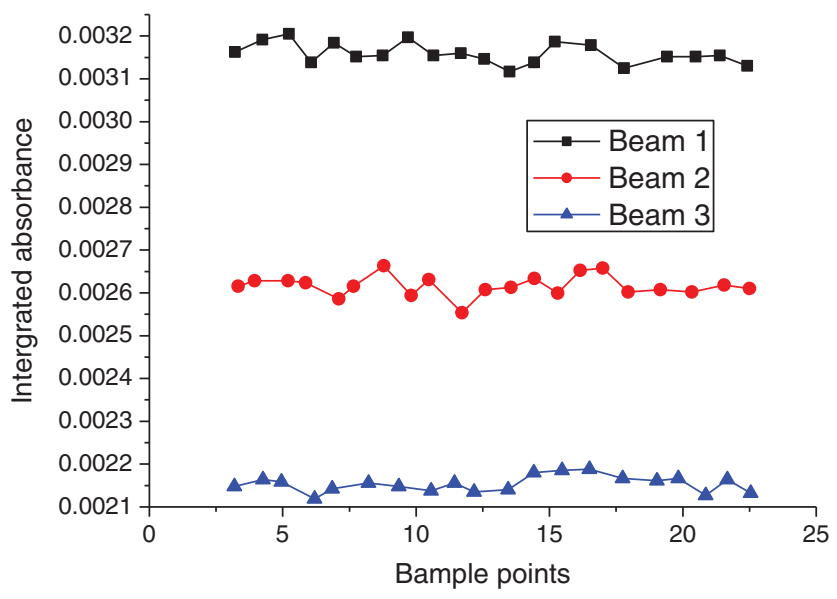

Figure 9: The trend of the projection value of the 3 signals continuously measured in the vertical direction before the rotation of the rotary table

The accurate values of reconstructed temperature and concentration are shown in Fig. 10. As shown in the data and trends in the figure, the TDLAS technology and CT-algebraic iterative reconstruction algorithm designed and discussed in this study can obtain clear and accurate measurement data for the vapor concentration and temperature in the flat flame furnace. The results are consistent with the real data laws. The temperature in the center of the furnace is relatively high, the temperature in the boundary area is relatively low, and the rate of change in the concentration is similar. By performing a two-dimensional reconstruction of the combustion heat transfer temperature and concentration on the surface of a flat flame furnace, the characteristics of the heat transfer can be more fully understood.
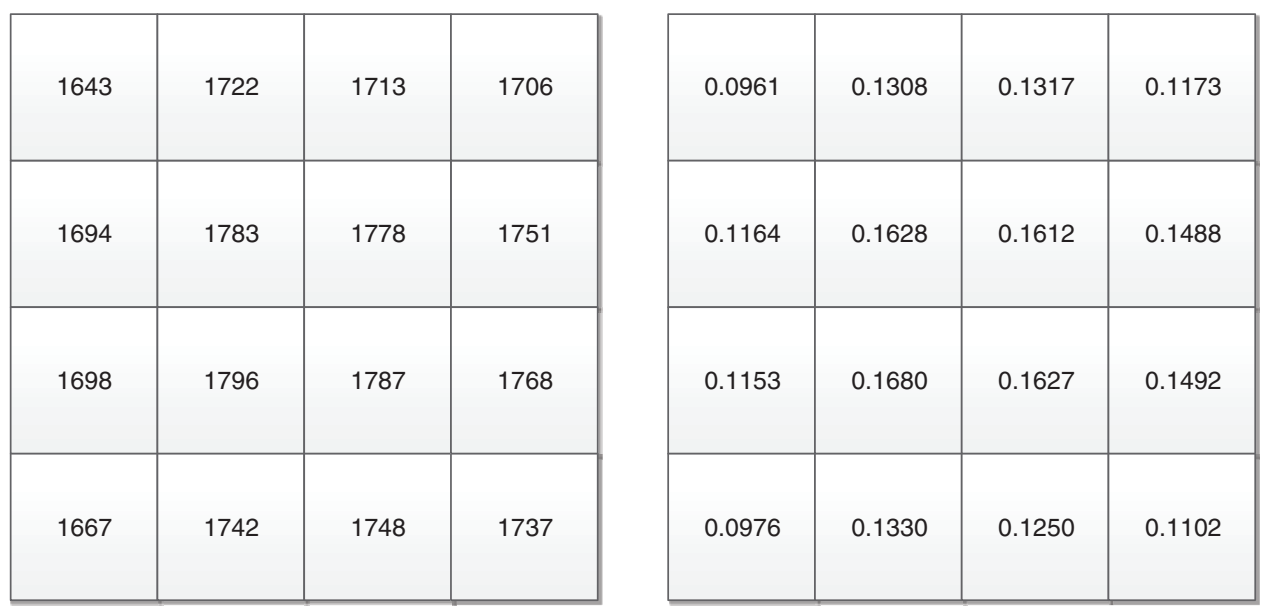

Figure 10: The accurate values of reconstructed temperature and concentration 


\section{Conclusion}

This study analyzes and explores the two-dimensional reconstruction of vapor concentration and furnace temperature in the surface combustion of the flat flame furnace by constructing a hybrid method based on the CT-algebraic iterative algorithm and TDLAS. The research results show that the two-dimensional reconstruction of the furnace temperature and vapor concentration of the flat flame furnace based on the hybrid method of CT-algebraic iterative algorithm and TDLAS has a significant impact. The analysis of the temperature and concentration through the monitoring of the optical path reveals basically consistent laws. The temperature and concentration in the central position are relatively high, while the temperature and concentration in the boundary area are relatively low. Besides, the transition from the center to the boundary is relatively uniform, while the temperature in the central area is relatively stable. This study also has some deficiencies in the research process, which are mainly caused by the fact that the conclusions obtained by the two-dimensional reconstruction simulation of furnace temperature and concentration for the hybrid method of CT-algebraic iterative algorithm and TDLAS are more from the experimental and theoretical stages. There will be many factors and problems of the operations under actual application conditions. Since this study is in the experimental stage, many external factors are ignored, and the results may be slightly less convincing. Nevertheless, this study has provided a useful reference for the investigation of the two-dimensional reconstruction of combustion qualitatively.

Funding Statement: The author(s) received no specific funding for this study.

Conflicts of Interest: The authors declare that they have no conflicts of interest to report regarding the present study.

\section{References}

1. Jiang, G., He, H., Yan, J., Xie, P. (2018). Multiscale convolutional neural networks for fault diagnosis of wind turbine gearbox. IEEE Transactions on Industrial Electronics, 66(4), 3196-3207. DOI 10.1109/ TIE.2018.2844805.

2. Ding, R., Zhou, X., Zhang, R., Lu, W. (2020). Research on the measurement and countermeasure of coal overcapacity in China: based on panel data of 25 provinces in China. Energy Engineering, 117(1), 27-39. DOI 10.32604/EE.2020.010418.

3. Peeters, C., Guillaume, P., Helsen, J. (2018). Vibration-based bearing fault detection for operations and maintenance cost reduction in wind energy. Renewable Energy, 116(PT.B), 74-87. DOI 10.1016/j. renene.2017.01.056.

4. Ziaja, A., Antoniadou, I., Barszcz, T., Staszewski, W. J., Worden, K. (2016). Fault detection in rolling element bearings using wavelet-based variance analysis and novelty detection. Journal of Vibration and Control, 22(2), 396-411. DOI 10.1177/1077546314532859.

5. Chen, J., Pan, J., Li, Z., Zi, Y., Chen, X. (2016). Generator bearing fault diagnosis for wind turbine via empirical wavelet transform using measured vibration signals. Renewable Energy, 89(6), 80-92. DOI 10.1016/j. renene.2015.12.010.

6. Jalali, H., Abbassi, H. (2020). Analysis of the influence of viscosity and thermal conductivity on heat transfer by $\mathrm{Al}_{2} \mathrm{O}_{3}$-water nanofluid. Fluid Dynamics \& Materials Processing, 16(2), 181-198. DOI 10.32604/ fdmp.2020.07804.

7. Cao, M. N., Qiu, Y. M., Feng, Y. H., Wang, H., Li, D. (2016). Study of wind turbine fault diagnosis based on unscented kalman filter and SCADA data. Energies, 9(10), 847. DOI 10.3390/en9100847.

8. Uma Maheswari, R. U., Umamaheswari, R. (2017). Trends in non-stationary signal processing techniques applied to vibration analysis of wind turbine drive train-a contemporary survey. Mechanical Systems and Signal Processing, 85(6), 296-311. DOI 10.1016/j.ymssp.2016.07.046.

9. Astolfi, D., Scappaticci, L., Terzi, L. (2017). Fault diagnosis of wind turbine gearboxes through temperature and vibration data. International Journal of Renewable Energy Research, 7(2), 965-976. 
10. Zhao, H., Liu, H., Hu, W., Yan, X. (2018). Anomaly detection and fault analysis of wind turbine components based on deep learning network. Renewable Energy, 127(8), 825-834. DOI 10.1016/j.renene.2018.05.024.

11. Zhang, W., Qiu, Y., Infield, D., Feng, Y., Sun, J. (2016). Applying thermophysics for wind turbine drivetrain fault diagnosis using SCADA data. IET Renewable Power Generation, 10(5), 661-668. DOI 10.1049/ietrpg.2015.0160.

12. Romero, A., Soua, S., Gan, T. H., Wang, B. (2018). Condition monitoring of a wind turbine drive train based on its power dependant vibrations. Renewable Energy, 123(10), 817-827. DOI 10.1016/j.renene.2017.07.086.

13. Kandukuri, S. T., Klausen, A., Karimi, H. R., Robbersmyr, K. G. (2016). A review of diagnostics and prognostics of low-speed machinery towards wind turbine farm-level health management. Renewable and Sustainable Energy Reviews, 53(13), 697-708. DOI 10.1016/j.rser.2015.08.061.

14. Bangalore, P., Letzgus, S., Karlsson, D., Patriksson, M. (2017). An artificial neural network-based condition monitoring method for wind turbines, with application to the monitoring of the gearbox. Wind Energy, 20(8), 1421-1438. DOI 10.1002/we.2102.

15. Hang, J., Zhang, J., Cheng, M. (2016). Application of multi-class fuzzy support vector machine classifier for fault diagnosis of wind turbine. Fuzzy Sets and Systems, 297(18), 128-140. DOI 10.1016/j.fss.2015.07.005.

16. Guo, J., Lu, S. L., Zhai, C., He, Q. B. (2018). Automatic bearing fault diagnosis of permanent magnet synchronous generators in wind turbines subjected to noise interference. Measurement Science and Technology, 29(2), 0250002.

17. Gonzalez, E., Gonzalez, E., Reder, M., Melero, J. J. (2016). SCADA alarms processing for wind turbine component failure detection. Journal of Physics: Conference Series, 753(7), 072019. DOI 10.1088/1742-6596/ 753/7/072019.

18. Chen, F., Fu, Z., Yang, Z. (2017). Research on intelligent fault identification technology of wind turbine supported by fault knowledge base. Modelling, Measurement and Control A, 90(1), 1-15. DOI 10.18280/mmc_a.900101.

19. Tautz-Weinert, J., Watson, S. J. (2017). Using SCADA data for wind turbine condition monitoring-a review. IET Renewable Power Generation, 11(4), 382-394. DOI 10.1049/iet-rpg.2016.0248.

20. Li, Z., Jiang, Y., Hu, C., Peng, Z. (2017). Difference equation based empirical mode decomposition with application to separation enhancement of multi-fault vibration signals. Journal of Difference Equations and Applications, 23(1-2), 457-467. DOI 10.1080/10236198.2016.1254206.

21. Jin, X., Gan, Y., Ju, W., Yang, X., Han, H. (2016). Research on wind turbine safety analysis: failure analysis, reliability analysis, and risk assessment. Environmental Progress \& Sustainable Energy, 35(6), 1848-1861. DOI 10.1002/ep.12413.

22. Georg, H., Matthias, R. (2018). Deep Learning for fault detection in wind turbines. Renewable and Sustainable Energy Reviews, 98(11), 189-198. DOI 10.1016/j.rser.2018.09.012.

23. Triveni, M., Panua, R. (2018). Numerical study of natural convection in a right triangular enclosure with sinusoidal hot wall and different configurations of cold walls. Fluid Dynamics and Materials Processing, 14(1), 1-21.

24. Dandani, M., Lepiller, V., Abderrahmane, G., Désévaux, P. (2018). Numerical visualizations of mixing enhancement in a 2D supersonic ejector. Fluid Dynamics and Materials Processing, 14(1), 23-37.

25. Neffah, Z., Kahalerras, H., Fersadou, B. (2018). Heat and mass transfer of a non-newtonian fluid flow in an anisotropic porous channel with chemical surface reaction. Fluid Dynamics and Materials Processing, 14(1), $39-56$. 